\title{
Study on Effect of Vitexicarpin on the Biological Behavior of Colorectal Cancer Cells by Circ_0000419/MicroRNA-224
}

\author{
YAO HUANG, Y. CAO ${ }^{1}$, P. BO, HONGPING ZHOU1, YAN WANG ${ }^{2}$ AND GUIMEI KONG ${ }^{3 *}$ \\ Medical school of Yangzhou University, Yangzhou 225009, Jiangsu, ${ }^{1}$ Department of Radiotherapy oncology, Nanjing Benq \\ medical center, Jiangsu, Nanjing 210019, '2Laboratory Department, Yangzhou Center for Disease Control and Prevention, \\ 3 Medical school of Yangzhou University, Yangzhou Center for Disease Control and Prevention \& Jiangsu Key Laboratory of \\ Zoonosis, Jiangsu, Yangzhou 225009, China
}

Huang et al: : Study on Effect of Vitexicarpin on the Biological Behavior

\begin{abstract}
To investigate the effect and molecular mechanism of vitexicarpin on the biological behavior of colorectal cancer cells is the objective of the study. The human epithelial cell line Caco-2 cells were divided into control group, low, middle and high dose groups of vitexicarpin, plasmid cloning DNA-circ_0000419 group, plasmid cloning DNA group, anti-microRNA-224 group, anti-microRNA-224-negative control group, vitexicarpin+small interfering-circ_0000419 group and vitexicarpin+microRNA-224 group. Cell activity was detected by 3-(4,5-dimethylthiazolyl-2)-2,5-diphenyltetrazolium bromide assay. Colony formation number was detected by plate clone formation experiment. Apoptosis was detected by flow cytometry. Scratch test was used to detect the migration distance of cells. Transwell method was used to detect the number of invasive cells; protein expression was detected by western blot; the expression levels of circ_0000419 and microRNA-224 were detected by real-time fluorescence quantitative polymerase chain reaction; dual-luciferase reporter assay was used to detect the targeting relationship between circ_0000419 and microRNA-224. After treatment with low, middle and high doses of vitexicarpin, the viability of Caco2 cells decreased, the number of colony formation decreased, the apoptosis rate increased, the migration distance decreased, the number of invasive cells decreased, the expression level of E-cadherin increased, the expression level of $\mathrm{N}$-cadherin decreased, the expression level of circ_0000419 increased and the expression level of microRNA-224 decreased $(\mathbf{p}<0.05)$. Overexpression of circ_0000419 or inhibition of miR-224 expression decreased Caco-2 cell viability, decreased colony formation, increased apoptosis rate, decreased cell migration distance, decreased invasive cell number, increased expression of E-cadherin and decreased expression of $\mathrm{N}$-cadherin $(\mathrm{p}<\mathbf{0 . 0 5})$. Interference with circ_0000419 or overexpression of microRNA-224 reverses the effects of vitexicarpin on proliferation, apoptosis, migration and invasion of Caco-2 cells; circ_0000419 targets the regulation of microRNA-224 expression. Vitexicarpin can inhibit the malignant biological behavior of colorectal cancer cells by circ_0000419/microRNA-224.
\end{abstract}

Key words: Vitexicarpin, circ_0000419, microRNA-224, real-time fluorescence quantitative polymerase chain reaction

Colorectal cancer is a common malignant tumor in the digestive system, which seriously endangers human health. It is found that Traditional Chinese medicine (TCM) has certain curative effect and value in anticolorectal cancer, so it is of great significance to develop more new TCM for its treatment ${ }^{[1,2]}$. Vitexicarpin/ Casticin, a methoxyl flavonoid extracted from Fructus Viticis, has an anti-tumor effect. It was found that vitexicarpin may block the cell cycle in Growth 2 Phase $\left(\mathrm{G}_{2}\right)$ /Mitosis (M) phase by inhibiting the expression of Cyclin-Dependent Kinase 1 (CDK1), Cellular Myelocytomatosis Oncogene (c-myc) and survivin, thereby inhibiting the proliferation and promoting the

*Address for correspondence E-mail: gmkong123456@163.com

September-October 2021 apoptosis of human non-small cell lung cancer cell line $(\mathrm{H} 322)^{[3]}$. Vitexicarpin also inhibits the growth of lung cancer xenografts in nude mice ${ }^{[4]}$. Vitexicarpin inhibits the growth of human prostate cancer cell line (PC-3) cells and promotes the apoptosis by blocking the cell cycle of prostate cancer PC-3 cells in $\mathrm{G}_{2} / \mathrm{M}$ phase ${ }^{[5]}$. Hedgehog signaling pathway inhibits Epithelial-to-Mesenchymal

This is an open access article distributed under the terms of the Creative
Commons Attribution-NonCommercial-ShareAlike 3.0 License, which
allows others to remix, tweak, and build upon the work non-commercially,
as long as the author is credited and the new creations are licensed under
the identical terms Accepted 06 September 2021 Revised 14 July 2021 Received 15 October 2019 Indian J Pharm Sci 2021;83(5):1007-1015 
Transition (EMT) in vitro by vitexicarpin, thereby reducing migration capacity of ovarian cancer cells ${ }^{[6]}$. However, the effect of vitexicarpin on the biological behavior of colorectal cancer cells and its mechanism are still unclear. MicroRNAs (miRNAs) affect the occurrence and development of colorectal cancer. As an important member of miRNA, miR-224 is significantly upregulated in colorectal cancer. Overexpression of miR-224 promotes the migration and invasion of colorectal cancer cells ${ }^{[7]}$. In addition, miR-224 mediates proliferation of human colon cancer cell line (HCT116) by targeting $\mathrm{Smad}_{4}^{[8]}$. Down regulation of miR224 significantly reduced HCT116 cell viability and promoted apoptosis by modulating Homeobox protein B3 (HoxB3) expression ${ }^{[9]}$. We found that circular RNA (circRNA) is capable of spongy regulation of miRNA and we predicted by online software that circ_0000419 binds to miR-224. Studies have reported significantly reduced levels of circ_0000419 in gastric cancer tissue and plasma and circ_0000419 may be involved in the development and progression of gastric cancer through its interaction with miRNA ${ }^{[10]}$. However, the effect of circ_0000419 on colorectal cancer cells and whether it modulates miR-224 is unknown. The purpose of this study is to investigate whether circ_0000419 and miR-224 are involved in the effects and mechanisms of vitexicarpin on the biological behavior of colorectal cancer cells.

\section{MATERIALS AND METHODS}

\section{Materials:}

Human epithelial cell line (Caco-2) cells were purchased from Wuxi Newgainbio Co., Ltd.; Minimum Essential Medium (MEM), culture medium was purchased from Shanghai Fusheng Industry Co., Ltd.; vitexicarpin (purity $>98 \%$ ) was purchased from Nanjing Dasf Biotechnology Co., Ltd.; 3-(4, 5-dimethylthiazolyl-2)-2, 5-diphenyltetrazolium bromide (MTT) kit and apoptosis detection kit were purchased from Dojindo Research Institute of Japan; Transwell Cabin and Matrigel were purchased from Corning Company of the United States; Radioimmunoprecipitation Assay (RIPA) protein lysate was purchased from Shanghai Bangyi Biotechnology Co., Ltd.; SYBR Premix ExTaq ${ }^{\mathrm{TM}}$ kit was purchased from Takara Company of Japan; dual-luciferase reporter gene detection kit was purchased from Shenzhen Zike Biological technology Co., Ltd.

\section{Treatment and grouping of cells:}

Caco-2 cells were cultured in MEM medium containing $10 \%$ fetal bovine serum and treated with $0.1 \mu \mathrm{mol} / 1$, $0.3 \mu \mathrm{mol} / 1$ and $1.0 \mu \mathrm{mol} / 1$ vitexicarpin as low, middle and high dose groups of vitexicarpin and those not treated were taken in control group. Circ_0000419 overexpression vector and Negative Control (NC), miR-224 inhibition expression vector and $\mathrm{NC}$ were transfected into Caco-2 cells, which were recorded as Plasmid Cloning DNA (pcDNA) circ_0000419 group, pcDNA group, anti-miR-224 group and anti-miR-NC group. Circ_0000419 inhibition expression vector or miR-224 overexpression vector were transfected into Caco- 2 cells and treated with $1.0 \mu \mathrm{mol} / 1$ vitexicarpin, designated as vitexicarpin+small interfering (si)circ_0000419 group and vitexicarpin+miR-224 group.

\section{MTT assay for cell proliferation activity:}

To the culture cells of each group after $48 \mathrm{~h}$, add $20 \mu \mathrm{l}$ MTT solution in each well, incubate for $4 \mathrm{~h}$, discard the supernatant, add $150 \mu$ Dimethylsulfoxide (DMSO) in each well, shake and react for $10 \mathrm{~min}$ and detect the absorbance (Optical Density (OD)) value at $490 \mathrm{~nm}$ with a microplate reader.

\section{Colony formation number by plate clone formation experiment:}

Take logarithmic growth phase cells of all groups, prepare cell suspension, inoculate into 6 well plate, culture for $2 \mathrm{w}$, then fix with methanol for $15 \mathrm{~min}$, then stain with Giemsa for $30 \mathrm{~min}$, count the colonies of $>50$ cells under light microscope.

\section{Apoptosis by flow cytometry:}

Collect cells in each group, add binding buffer to resuspend cells after rinsing, then add $10 \mu \mathrm{l}$ of Annexin V-FITC and $5 \mu$ of Propidium Iodide (PI), mix well and incubate in the dark for $10 \mathrm{~min}$; detect the apoptosis rate on the apparatus.

\section{Cell migration distance measured by scratch test:}

After digesting the cells in each group, $5 \times 10^{5}$ cells/well were inoculated and cultured in culture plate. After the cells were spread out, they were scratched with tip to make certain distance between cells. After washing with Phosphate Buffered Saline (PBS), the cells were continuously cultured. Photographs were taken at $0 \mathrm{~h}$ and $24 \mathrm{~h}$, respectively. The migration distances of cells in each group were measured by Imageplus software. 


\section{Transwell assay for the detection of invasive cells:}

Each group was cultured for $48 \mathrm{~h}$ and resuspended after digestion. Transwell chamber was plated with Matrigel, inoculated with cells and cultured for $24 \mathrm{~h}$. After removal, residual cells were wiped off with a cotton swab, fixed with formaldehyde for $10 \mathrm{~min}$ and stained with $0.1 \%$ crystal violet for $10 \mathrm{~min}$. Microscopic observation was performed and it was photographed and counted. Mean value of 5 visual fields was calculated for each group.

\section{Protein expression by western blot:}

Total protein in each group of cells was extracted, electrophoresed by Sodium Dodecyl-Sulfate Polyacrylamide Gel Electrophoresis (SDS-PAGE), then transferred to Polyvinylidene Fluoride (PVDF) membrane by wet rotation method, blocked in $5 \%$ skim milk solution for $1.5 \mathrm{~h}$, first added primary antibody at $4^{\circ}$ for overnight, then added secondary antibody at room temperature for $2 \mathrm{~h}$, developed by Electrochemiluminescence (ECL), analyzed the gray value of protein band and calculated the relative expression amount of protein.

\section{Expression levels of circ_0000419 and miR-224 by Real-Time Fluorescence Quantitative Polymerase Chain Reaction (RT-qPCR):}

The total DNA of each group of cells was extracted, the Complementary DNA (cDNA) was synthesized and amplified by RT-PCR at $95^{\circ}$ for $5 \mathrm{~min}, 95^{\circ}$ for $30 \mathrm{~s}, 60^{\circ}$ for $30 \mathrm{~s}$ and $72^{\circ}$ for $30 \mathrm{~s}$ for 40 cycles and the relative expression amount was calculated by $2^{-\Delta \Delta \mathrm{Ct}}$ method. Using Glyceraldehyde 3-Phosphate Dehydrogenase (GAPDH) and U6 as internal parameters, the upstream primer sequence of circ_0000419 was 5'-AAGTCGGAAGCAGCTCACAA-3' and the downstream primer sequence was 5 ' - G T T T C A C C G A A A C C T C C C G A - 3 '; GAPDH upstream primer sequence: 5 ' - T G T T G C C A T C A A T C A C C C C T T - 3 ', downstream primer sequence: 5 ' - C T C C A C C G A C G T A C T C A G C G - 3 '; miR-224 upstream primer sequence: 5 ' - G C G A G G T C A A G T C A C TA G T G G T-3 ', downstream primer sequence: 5'-CGAGAAGCTTGCATCACCAGAGAACG-3'; U6 upstream primer sequence: 5 ' - C T C G C T T C G G C A G C A C ATATA C T-3', downstream primer sequence: 5'-ACGCTTCACGAATTTGCGTGTC-3'; the primer was synthesized by Shanghai Sangon Biotech Co., Ltd.
Dual-luciferase reporter assay:

The Wild Type (wt) and Mutant (mut) luciferase vectors of circ_0000419 were constructed and cotransfected into Caco-2 cells with miR-NC and miR224 , respectively and cell luciferase activity was tested as described in the kit.

\section{Statistical analysis:}

Statistical Package for the Social Sciences (SPSS) 20.0 software was used for statistical analysis. The measurement data conforming to normal distribution were expressed as mean \pm Standard Deviation (SD) $(\overline{\mathrm{x}} \pm \mathrm{s}), \mathrm{t}$ test was performed for comparison between two groups. One-way analysis of variance was used for comparison among multiple groups, $\mathrm{p}<0.05$ was used as statistical significance.

\section{RESULTS AND DISCUSSION}

Effects of vitexicarpin on proliferation and apoptosis of Caco-2 cells were described below. Compared with the control group, the activity of Caco- 2 cells decreased in low, middle and high dose groups of vitexicarpin, the colony formation number decreased and the apoptosis rate increased $(\mathrm{p}<0.05)$ (fig. 1 and Table 1).

Effect of vitexicarpin on migration and invasion of Caco-2 cells were given below. Compared with the control group, the migration distance of Caco-2 cells decreased, the number of invasive cells decreased, the expression level of E-cadherin increased and the expression level of $\mathrm{N}$-cadherin decreased in the low, middle and high dose groups of vitexicarpin $(\mathrm{p}<0.05)$ (fig. 2 and Table 2).

Effect of vitexicarpin on the expression of circ_0000419 and miR-224 in Caco-2 cells were shown below. Circ_0000419 expression in Caco-2 cells was increased and miR-224 expression was decreased in the low, middle and high dose groups of vitexicarpin compared with control group $(\mathrm{p}<0.05)$ (Table 3$)$.

Effect of circ_0000419 on the migration and invasion of Caco-2 proliferation and apoptosis is explained below. Compared with the pcDNA group, the pcDNAcirc_0000419 group had higher levels of circ_0000419 expression, lower levels of miR-224 expression, lower Caco-2 cell viability, lower numbers of colony formation, higher rates of apoptosis, lower cell migration distances, lower numbers of invasive cells, higher levels of E-cadherin expression and lower levels of $\mathrm{N}$-cadherin expression $(\mathrm{p}<0.05)$ (fig. 3 and Table 4). 
A
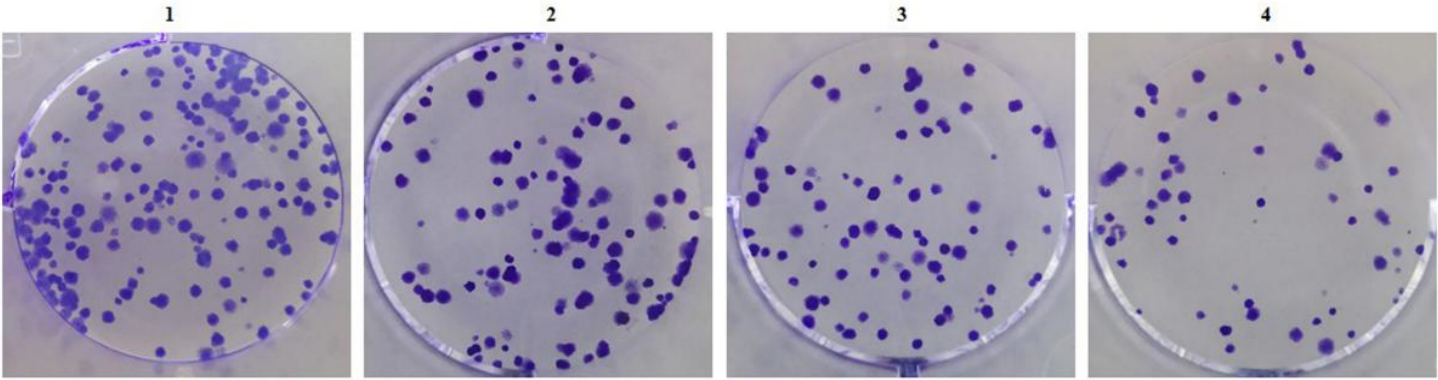

B
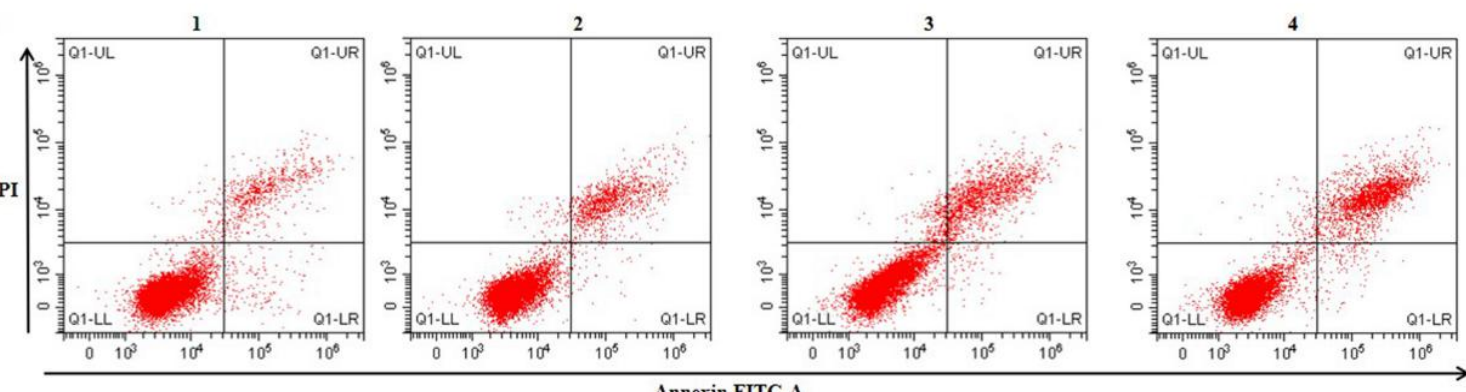

Fig. 1: The effect of vitexicarpin on Caco-2 colony formation and apoptosis, (1) Control; (2) low dose of vitexicarpin; (3) middle dose of vitexicarpin; (4) high dose of vitexicarpin

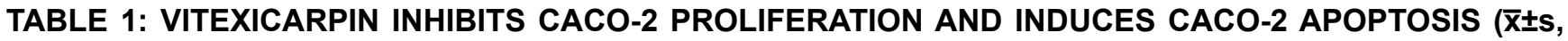
$\mathrm{n}=3$ )

\begin{tabular}{lccc}
\hline Grouping & OD value & Number of colony formation (nos.) & Apoptosis rate (\%) \\
\hline Control & $1.34 \pm 0.09$ & $134.33 \pm 6.24$ & $7.02 \pm 0.36$ \\
Low dose of vitexicarpin & $1.10 \pm 0.06^{*}$ & $107.00 \pm 5.72^{*}$ & $11.59 \pm 0.47^{*}$ \\
Middle dose of vitexicarpin & $0.84 \pm 0.05^{*}$ & $76.67 \pm 4.64^{*}$ & $15.86 \pm 0.85^{*}$ \\
High dose of vitexicarpin & $0.57 \pm 0.03^{*}$ & $53.67 \pm 2.49^{*}$ & $22.33 \pm 0.99^{*}$ \\
F & 87.543 & 149.626 & 247.853 \\
p & 0.000 & 0.000 & 0.000 \\
\hline
\end{tabular}

Note: Compared with control group, ${ }^{*} \mathrm{p}<0.05$

A
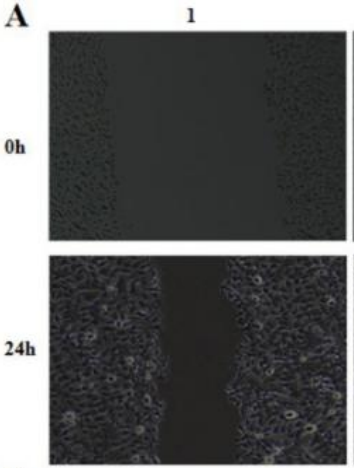

B
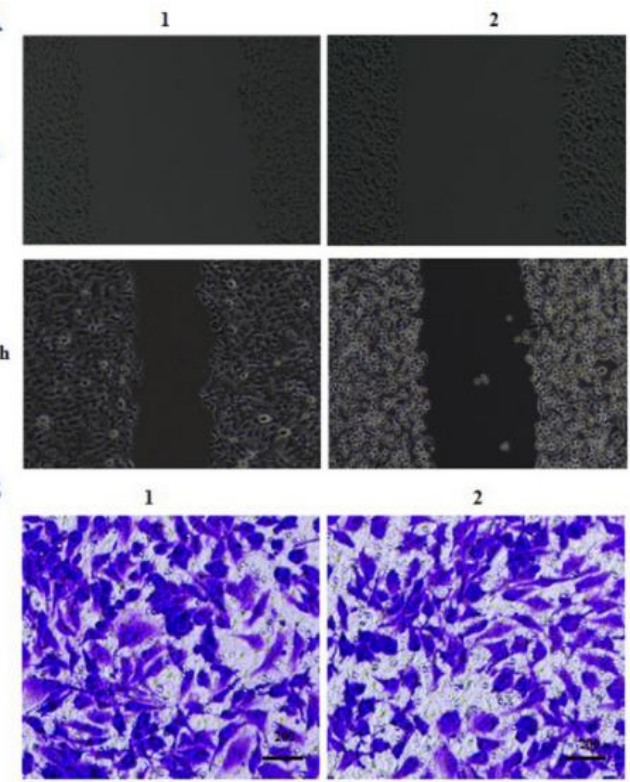
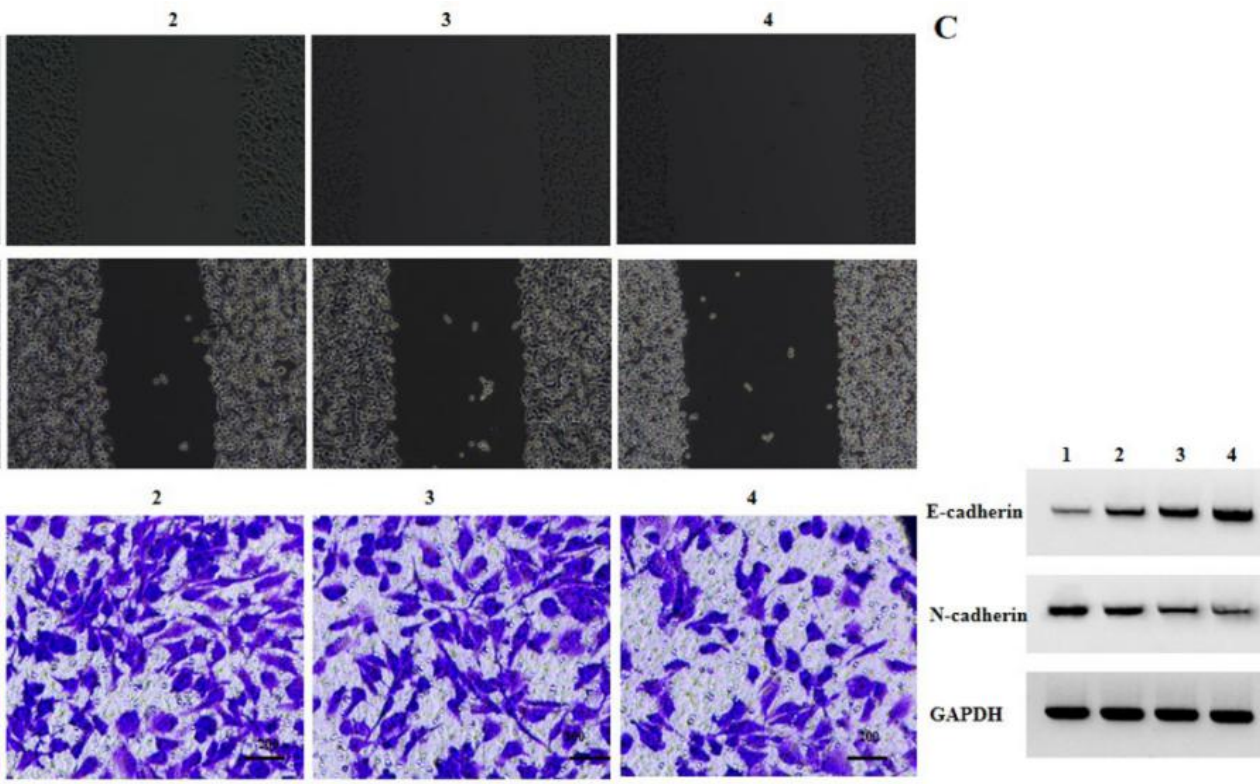

Fig. 2: The effect of vitexicarpin on the migration distance of Caco-2, the number of invasive cells and the expression of E-cadherin and N-cadherin, (1) Control; (2) low dose of vitexicarpin; (3) middle dose of vitexicarpin; (4) high dose of vitexicarpin 
TABLE 2: VITEXICARPIN INHIBITS CACO-2 MIGRATION AND INVASION ( $\overline{\mathbf{x}} \pm \mathbf{s}, \mathbf{n}=\mathbf{3})$

\begin{tabular}{lcccc}
\hline Grouping & Migration distance $(\mu \mathrm{m})$ & Number of invasive cells $($ nos.) & E-cadherin & $\mathrm{N}$-cadherin \\
\hline Control & $185.75 \pm 7.49$ & $155.00 \pm 5.72$ & $0.14 \pm 0.02$ & $0.75 \pm 0.06$ \\
Low dose of vitexicarpin & $149.84 \pm 5.60^{*}$ & $129.33 \pm 4.50^{*}$ & $0.36 \pm 0.03^{*}$ & $0.46 \pm 0.04^{*}$ \\
Middle dose of vitexicarpin & $114.82 \pm 5.16^{*}$ & $97.33 \pm 3.30^{*}$ & $0.58 \pm 0.05^{*}$ & $0.27 \pm 0.03^{*}$ \\
High dose of vitexicarpin & $83.31 \pm 3.54^{*}$ & $65.33 \pm 2.05^{*}$ & $0.80 \pm 0.06^{*}$ & $0.13 \pm 0.01^{*}$ \\
F & 185.283 & 266.959 & 130.811 & 139.274 \\
p & 0.000 & 0.000 & 0.000 & 0.000 \\
\hline
\end{tabular}

Note: Compared with control group, ${ }^{*} \mathrm{p}<0.05$

TABLE 3: DETECTION OF CIRC_0000419 and miR-224 EXPRESSION ( $\overline{\mathbf{x}} \pm \mathbf{s}, \mathrm{n}=3$ )

\begin{tabular}{lcc}
\hline Grouping & Circ_0000419 & miR-224 \\
\hline Control & $1.00 \pm 0.00$ & $1.00 \pm 0.00$ \\
Low dose of vitexicarpin & $1.65 \pm 0.07^{*}$ & $0.78 \pm 0.05^{*}$ \\
Middle dose of vitexicarpin & $2.28 \pm 0.09^{*}$ & $0.47 \pm 0.04^{*}$ \\
High dose of vitexicarpin & $4.19 \pm 0.13^{*}$ & $0.19 \pm 0.02^{*}$ \\
F & 760.321 & 335.111 \\
p & 0.000 & 0.000 \\
\hline
\end{tabular}

Note: Compared with control group, ${ }^{*} \mathrm{p}<0.05$

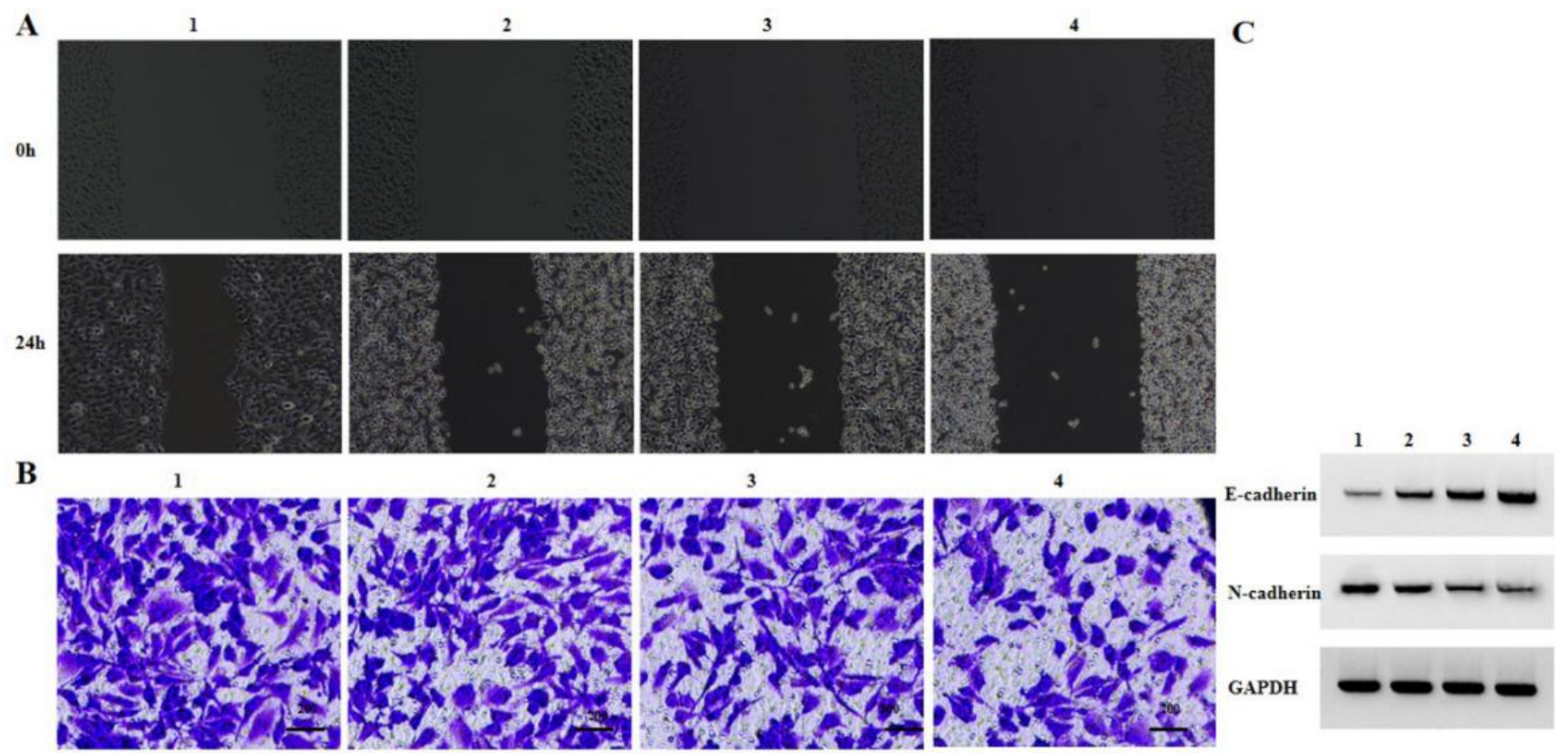

Fig. 3: The effect of circ_0000419 on Caco-2 colony formation, apoptosis, migration distance, number of invasive cells and expression of E-cadherin and N-cadherin

TABLE 4: CIRC_0000419 INHIBITS CACO-2 PROLIFERATION, MIGRATION AND INVASION AND INDUCES CACO-2 APOPTOSIS ( $\overline{\mathrm{x}} \pm \mathbf{s}, \mathrm{n}=3$ )

\begin{tabular}{|c|c|c|c|c|c|c|c|c|c|}
\hline Grouping & $\begin{array}{c}\text { Circ } \\
\_0000419\end{array}$ & miR-224 & OD value & $\begin{array}{l}\text { Number } \\
\text { of colony } \\
\text { formation } \\
\text { (nos.) }\end{array}$ & $\begin{array}{l}\text { Apoptosis } \\
\text { rate (\%) }\end{array}$ & $\begin{array}{c}\text { Migration } \\
\text { distance } \\
(\mu \mathrm{m})\end{array}$ & $\begin{array}{l}\text { Number of } \\
\text { invasive } \\
\text { cells (nos.) }\end{array}$ & $\frac{\text { E }}{\text {-cadherin }}$ & $\stackrel{N}{N}$ \\
\hline pcDNA & $1.00 \pm 0.00$ & $1.00 \pm 0.00$ & $1.34 \pm 0.09$ & $134.67 \pm 7.13$ & $7.04 \pm 0.46$ & $186.03 \pm 7.92$ & $155.67 \pm 6.60$ & $0.14 \pm 0.01$ & $0.76 \pm 0.06$ \\
\hline $\begin{array}{l}\text { pcDNA- } \\
\text { circ_0000419 }\end{array}$ & $3.85 \pm 0.07^{*}$ & $0.23 \pm 0.02^{*}$ & $0.66 \pm 0.03^{*}$ & $59.33 \pm 3.40^{*}$ & $20.47 \pm 0.94^{*}$ & $97.18 \pm 4.12^{*}$ & $74.33 \pm 2.62^{*}$ & $0.68 \pm 0.06^{*}$ & $0.23 \pm 0.02^{*}$ \\
\hline $\mathrm{t}$ & 4.924 & 66.684 & 12.415 & 16.250 & 22.227 & 17.238 & 19.840 & 15.376 & 14.515 \\
\hline $\mathrm{p}$ & 0.000 & 0.000 & 0.000 & 0.000 & 0.000 & 0.000 & 0.000 & 0.000 & 0.000 \\
\hline
\end{tabular}

Note: Compared with pcDNA group, ${ }^{*} \mathrm{p}<0.05$ 
Effect of miR-224 inhibition on migration and invasion of Caco-2 proliferation and apoptosis is given below. Compared with the anti-miR-NC group, the antimiR-224 group had lower levels of miR-224 expression, lower levels of Caco-2 cell viability, lower numbers of colony formation, higher rates of apoptosis, lower cell migration distances, lower numbers of invasive cells, higher levels of E-cadherin expression and lower levels of N-cadherin expression ( $p<0.05)$ (fig. 4 and Table 5).

Verification of target relationship between circ_0000419 and miR-224 is explained here. The circ_0000419 and miR-224 had complementary sequences (fig. 5); the luciferase activity of cells co-transfected with wtcirc_0000419 and miR-224 was lower than that of Caco-2 cells co-transfected with wt-circ_0000419 and miR-NC ( $\mathrm{p}<0.05)$; while the luciferase activity of cells co-transfected with mut-circ_0000419 and miR-224 or miR-NC was not significantly different (Table 6).

Effect of interference with circ_0000419 or overexpression of miR-224 on proliferation, apoptosis and migration of Caco-2 induced by vitexicarpin is shown below. Compared with vitexicarpin group, vitexicarpin+si-circ_0000419 group and vitexicarpin+miR-224 group had higher expression level of miR-224, higher activity of Caco-2 cells, higher number of colony formation, lower apoptosis rate, longer migration distance, higher number of invasive cells, lower expression level of E-cadherin and higher expression level of $\mathrm{N}$-cadherin $(\mathrm{p}<0.05)$ (fig. 6, Table 7 and Table 8).

Recent studies have shown that TCM has unique advantages in the adjuvant treatment of colorectal cancer, can improve the prognosis of patients with colorectal cancer and improve the quality of life of patients and plays an important role in the treatment of colon cancer ${ }^{[11,12]}$. It has been reported that vitexicarpin inhibits rat pituitary tumor cell line (GH3), pituitary adenoma cell proliferation through mitochondria-mediated apoptosis ${ }^{[13]}$. Vitexicarpin inhibits cell proliferation and reduces gastric cell migration and invasion by downregulating the expression of $\mathrm{G}$ protein subunit alpha $\mathrm{O} 1$

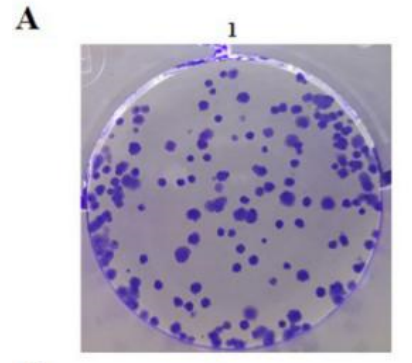

B

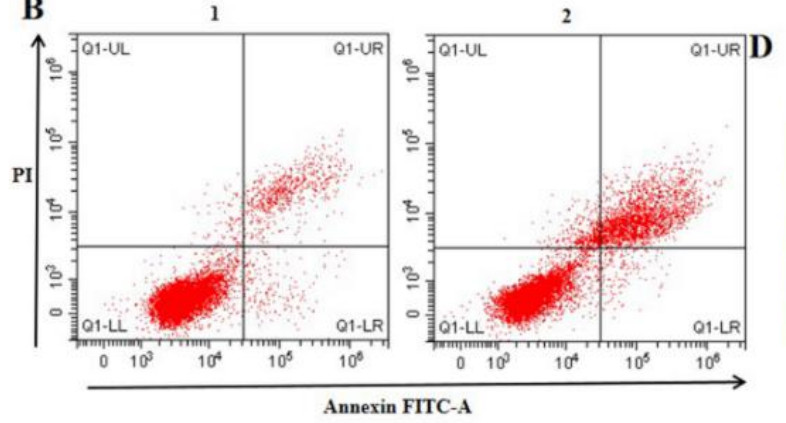

C

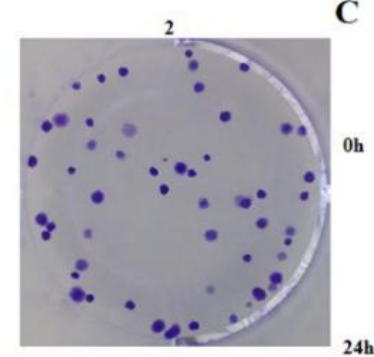

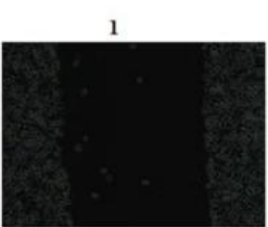

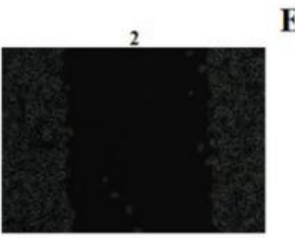

$\mathbf{E}$
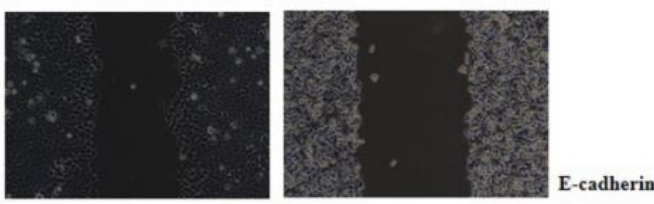

1
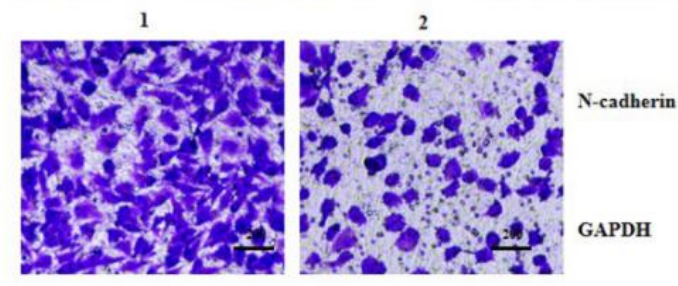

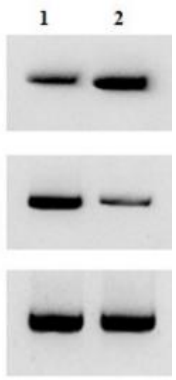

Fig. 4: The effect of inhibiting miR-224 on Caco-2 colony formation, apoptosis, migration distance, number of invasive cells and expression of E-cadherin and N-cadherin

TABLE 5: INHIBITION OF miR-224 CAN INHIBIT CACO-2 PROLIFERATION, MIGRATION AND INVASION AND INDUCE CACO-2 APOPTOSIS ( $\bar{x} \pm s, n=3)$

\begin{tabular}{lcccccccc}
\hline Grouping & miR-224 & OD value & $\begin{array}{c}\text { Number } \\
\text { of colony } \\
\text { formation } \\
(\text { nos.) }\end{array}$ & $\begin{array}{c}\text { Apoptosis } \\
\text { rate }(\%)\end{array}$ & $\begin{array}{c}\text { Migration } \\
\text { distance } \\
(\mu \mathrm{m})\end{array}$ & $\begin{array}{c}\text { Number of } \\
\text { invasive } \\
\text { cells (nos.) }\end{array}$ & E-cadherin & N-cadherin \\
\hline Anti-miR-NC & $1.00 \pm 0.00$ & $1.36 \pm 0.09$ & $134.33 \pm 6.02$ & $7.04 \pm 0.47$ & $185.49 \pm 9.99$ & $154.67 \pm 7.41$ & $0.13 \pm 0.02$ & $0.75 \pm 0.07$ \\
Anti-miR-224 & $0.12 \pm 0.01^{*}$ & $0.53 \pm 0.03^{*}$ & $45.00 \pm 1.63^{*}$ & $25.03 \pm 1.03^{*}$ & $74.52 \pm 2.71^{*}$ & $54.33 \pm 1.70^{*}$ & $0.89 \pm 0.06^{*}$ & $0.05 \pm 0.02^{*}$ \\
$t$ & 152.420 & 15.154 & 24.808 & 27.522 & 18.569 & 22.860 & 20.813 & 16.654 \\
p & 0.000 & 0.000 & 0.000 & 0.000 & 0.000 & 0.000 & 0.000 & 0.000 \\
\hline
\end{tabular}

Note: Compared with anti-miR-NC group, ${ }^{*} \mathrm{p}<0.05$ 


\section{wt-circ_0000419 5' GCUGACUUAU 3' \\ miR-224 3' UUGCCUUGGUGAUCACUGAAC $\quad 5^{\prime}$ \\ mut-circ_0000419 5' GCGACAAGU 3'}

Fig. 5: The complementary sequence of circ_0000419 and miR-224

TABLE 6: DUAL-LUCIFERASE REPORTER ASSAY ( $\overline{\mathbf{x}} \pm \mathbf{s}, \mathrm{n}=3)$

\begin{tabular}{lcc}
\hline Group & Wt-circ_0000419 & Mut-circ_0000419 \\
\hline miR-NC & $1.01 \pm 0.10$ & $0.99 \pm 0.08$ \\
miR-224 & $0.36 \pm 0.03^{*}$ & $0.93 \pm 0.06$ \\
$\mathrm{t}$ & 10.784 & 1.039 \\
$\mathrm{p}$ & 0.000 & 0.357 \\
\hline
\end{tabular}

Note: Compared with miR-NC group, ${ }^{*} \mathrm{p}<0.05$

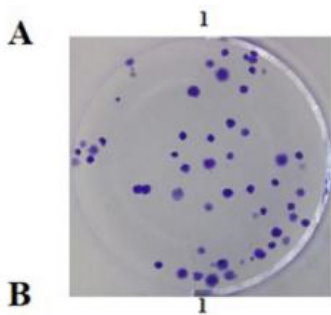

B

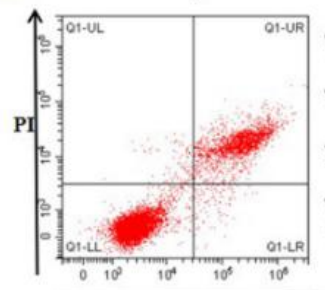

D
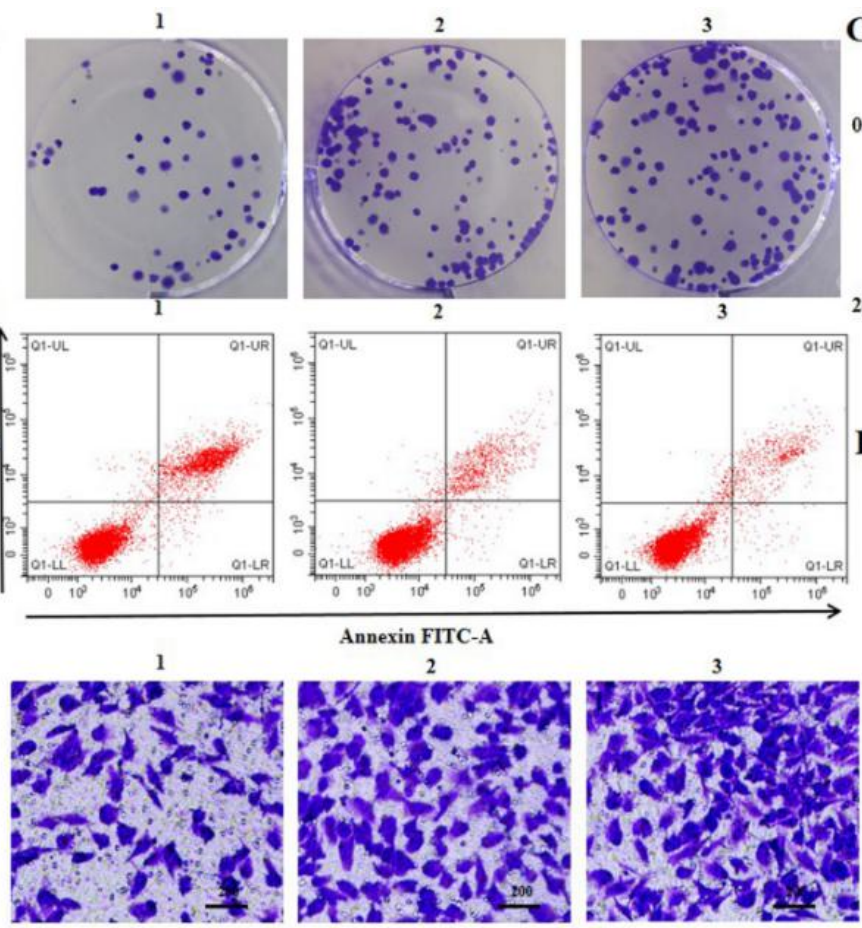
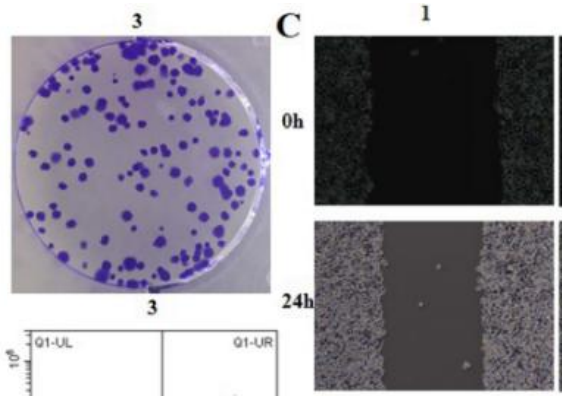

E

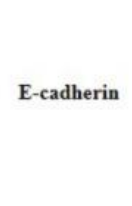

N-cadherin
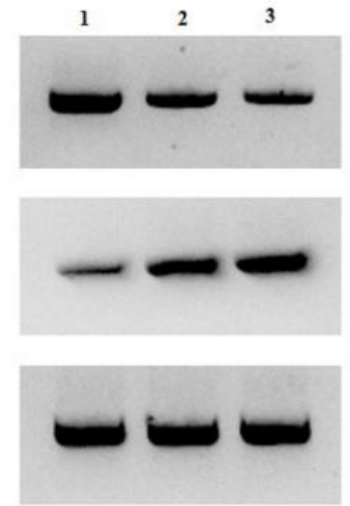

Fig. 6: The effect of interference with circ_0000419 or overexpression of miR-224 on Caco-2 colony formation, apoptosis, migration distance, number of invasive cells and the expression of $\mathbf{E}$-cadherin and $\mathrm{N}$-cadherin induced by vitexicarpin

TABLE 7: INTERFERENCE WITH CIRC_0000419 OR OVEREXPRESSION OF miR-22 CAN REVERSE THE EFFECT OF VITEXICARPINE ON THE PROLIFERATION AND APOPTOSIS OF CACO-2 $(\bar{x} \pm s, n=3)$

\begin{tabular}{lccccc}
\hline Grouping & Circ_0000419 & miR-224 & OD value & $\begin{array}{c}\text { Number of colony } \\
\text { formation (nos.) }\end{array}$ & $\begin{array}{c}\text { Apoptosis rate } \\
\text { (\%) }\end{array}$ \\
\hline Vitexicarpin & $4.20 \pm 0.16$ & $0.19 \pm 0.03$ & $0.57 \pm 0.04$ & $53.67 \pm 2.36$ & $22.38 \pm 1.00$ \\
Vitexicarpin+si-circ_0000419 & $1.28 \pm 0.07^{*}$ & $0.84 \pm 0.05^{*}$ & $1.15 \pm 0.06^{*}$ & $115.33 \pm 4.92^{*}$ & $9.48 \pm 0.63^{*}$ \\
Vitexicarpin+miR-224 & - & $0.93 \pm 0.05^{*}$ & $1.25 \pm 0.08^{*}$ & $125.67 \pm 5.31^{*}$ & $7.77 \pm 0.32^{*}$ \\
F(t) & 28.960 & 248.695 & 104.586 & 235.274 & 382.965 \\
$\mathrm{p}$ & 0.000 & 0.000 & 0.000 & 0.000 & 0.000 \\
\hline
\end{tabular}

Note: Compared with the vitexicarpin group, the ${ }^{*} \mathrm{p}<0.05$

(GNAO1) ${ }^{[14]}$. Vitexicarpin inhibits esophageal cancer cell proliferation and promotes apoptosis by modulating mitochondrial apoptosis and c-Jun N-terminal kinase
(JNK) signaling pathways ${ }^{[15]}$. Vitexicarpin impairs the growth of oral cancer cellosaurus cell line (SCC-4) cells and induces apoptosis through cell cycle arrest ${ }^{[16]}$. 
TABLE 8: INTERFERENCE WITH CIRC_0000419 OR OVEREXPRESSION OF miR-22 CAN REVERSE THE EFFECT OF VITEXICARPIN ON THE MIGRATION AND INVASION OF CACO-2 $(\overline{\mathrm{x}} \pm \mathbf{s}, \mathrm{n}=3)$

\begin{tabular}{lcccc}
\hline Grouping & Migration distance $(\mu \mathrm{m})$ & Number of invasive cells $($ nos.) & E-cadherin & N-cadherin \\
\hline Vitexicarpin & $84.03 \pm 4.12$ & $64.67 \pm 1.70$ & $0.80 \pm 0.07$ & $0.14 \pm 0.01$ \\
Vitexicarpin+si-circ_0000419 & $160.46 \pm 5.11^{*}$ & $136.67 \pm 4.19^{*}$ & $0.24 \pm 0.02^{*}$ & $0.57 \pm 0.05^{*}$ \\
Vitexicarpin+miR-224 & $177.60 \pm 5.95^{*}$ & $148.67 \pm 5.31^{*}$ & $0.18 \pm 0.01^{*}$ & $0.66 \pm 0.06^{*}$ \\
F & 284.575 & 381.891 & 194.889 & 112.113 \\
p & 0.000 & 0.000 & 0.000 & 0.000 \\
\hline
\end{tabular}

Note: Compared with the vitexicarpin group, the ${ }^{*} \mathrm{p}<0.05$

The above studies indicate that vitexicarpin inhibits the malignant biological behavior of a variety of tumors. In this study, after treatment of Caco-2 cells with different concentrations of Vitein decreased cell viability, the results showed increased apoptosis rate, decreased colony formation and number of invasive cells, decreased cell migration distance, increased expression of E-cadherin and decreased expression of N-cadherin, indicating that vitexicarpin inhibits the proliferation, migration and invasion of Caco- 2 cells and promotes the apoptosis of cells.

Inhibition of miR-224 has been reported to reduce colorectal cancer cell proliferation, enhance apoptosis and reduce doxorubicin resistance in cellosaurus cell line SW80 cells ${ }^{[17]}$. Inhibition of miR-224 inhibits proliferation, migration and invasion of cervical cancer cells by targeting pentraxin-related protein PTX ${ }^{[18]}$. Here we show that inhibition of miR-224 expression decreases Caco-2 cell viability, increases apoptosis rate, decreases colony formation and number of invasive cells, decreases cell migration distance, increases E-cadherin expression and decreases $\mathrm{N}$-cadherin expression, indicating that inhibition of miR-224 expression inhibits Caco-2 cell proliferation, migration and invasion and promotes apoptosis, which is consistent with previous studies of the effects of miR224 on colorectal cancer.

Furthermore, it has been reported that circ-ITCH [E3 ubiquitin-protein ligase] inhibits the proliferation, migration and invasion of bladder cancer by sponging miR-17/miR-224 and regulating the expression of cyclin-dependent kinase inhibitor $\mathrm{p} 21$ and Phosphatase and Tensin Homolog (PTEN) ${ }^{[19]}$. Circ_0004872 inhibits gastric cancer progression by modulating miR-224[20]. These results suggest that circRNA may influence tumor progression by modulating miR-224. Our results show that circ_0000419 targets the regulation of miR-224 expression. To investigate the effect of circ_0000419 on the biological behavior of colorectal cancer, we overexpressed circ_0000419. The results showed that the activity of Caco-2 cells decreased, the rate of apoptosis increased, the number of colony formation and the number of invasive cells decreased, the migration distance decreased, the expression level of E-cadherin increased and the expression level of $\mathrm{N}$-cadherin decreased, indicating that overexpression of circ_0000419 inhibited the proliferation, migration and invasion of Caco-2 cells and promoted the apoptosis of Caco-2 cells. Gardenoside has been reported to promote apoptosis by down regulating miR-224, which inhibits proliferation, migration and invasion of human liver cancer cell line HepG2 and cellosaurus cell line Huh-7 cells ${ }^{[21]}$. Our results suggest that vitexicarpin increases the expression of circ_0000419 and decreases the expression of miR-224, whereas interference with circ_0000419 or overexpression of miR-224 reverses the effects of vitexicarpin on proliferation, apoptosis, migration and invasion of Caco-2 cells.

In conclusion, vitexicarpin can inhibit the malignant biological behavior of colorectal cancer cells by circ_0000419/miR-224.

\section{Funding:}

The work was supported by National Natural Science Foundation of China (8167140158, 81272537), the Open Project Program of Jiangsu Key Laboratory of Zoonosis (No. R2001, R2002). This work was supported by a grant (SRD20180141) funded by National key clinical sub-project of Benq hospital.

\section{Conflicts of interest:}

The authors declared no conflict of interest.

\section{REFERENCES}

1. Zhang K, Li CG. Basic research progress of traditional Chinese medicine in the treatment of colon cancer. Int $\mathrm{J}$ Tradit Chin Med 2020;4(9):936-8.

2. Wang L, Li H, Li J. Advances in the application of traditional Chinese medicine in the treatment of colorectal cancer. Mod Digest Interv Diagn Treat 2020;25(9):1262-4.

3. Li S, Lei G, Ma X. Effects and mechanisms of vitexicarpin on human non-small cell lung cancer cell line H322. Mod Med Oncol 2020;28(7):1072-6.

4. Zhang J. Growth inhibition and its mechanism of vitexicarpin 
on nude mice bearing lung cancer. Shaanxi J Tradit Chin Med; 2017.

5. Meng F. Apoptosis and G2/M arrest of human prostate cancer PC-3 cells induced by vitexicarpin. Liaoning Norm Univ; 2013.

6. Zhang J, Cui Y, Sun S, Cao J, Fang X. Casticin inhibits the epithelial-mesenchymal transition in ovarian carcinoma via the hedgehog signaling pathway. Oncol lett 2018;15(4):4495-502.

7. Zheng Q, Jane JY, Li C, Li J, Wang J, Wang S. miR-224 targets $\mathrm{BTRC}$ and promotes cell migration and invasion in colorectal cancer. 3 Biotech 2020;10(11):1-10.

8. Zhou J, Hu M, Wang F, Song M, Huang Q, Ge B. miR-224 controls human colorectal cancer cell line HCT116 proliferation by targeting Smad4. Int J Med Sci 2017;14(10):937-42.

9. Li QC, Xue JH, Li W, Xing GQ. Effects of miR-224 on proliferation and apoptosis of colorectal cancer cells by regulating the expression of homeobox gene B3. J Clin Med Pract 2018;22(17):5-10.

10. Tao X, Shao Y, Lu R, Ye Q, Xiao B, Ye G, et al. Clinical significance of hsa_circ_0000419 in gastric cancer screening and prognosis estimation. Pathol Res Pract 2020;216(1):152763.

11. Lin Y, Ma L, Bulibu J, Kebinu M. Application of traditional Chinese medicine in the adjuvant treatment of colon cancer. China Pharm 2018;21(9):1637-40.

12. Yang Y. Advantages and prospects of traditional Chinese medicine in the treatment of colorectal cancer. Chin J Integr Med 2020;40(11):14-7.

13. Ying G, Chen G. Study on the Inhibitory effect of vitexicarpin on the proliferation of pituitary GH3 cells. Zhejiang J Integr Tradit Chin West Med 2015;25(3):233-6.
14. Wang SP, Yu L, Xie J, Liu XM, Li H. Vitexicarpin inhibits overexpression of GNAO1 and plays a role in gastric cancer cell proliferation and apoptosis. Bangladesh $\mathrm{J}$ Pharmacol 2015;10(1):63-8.

15. Qiao Z, Cheng Y, Liu S, Ma Z, Li S, Zhang W. Casticin inhibits esophageal cancer cell proliferation and promotes apoptosis by regulating mitochondrial apoptotic and JNK signaling pathways. Naunyn Schmiedebergs Arch Pharmacol 2019;392(2):177-87.

16. Chou GL, Peng SF, Liao CL, Ho HC, Lu KW, Lien JC, et al. Casticin impairs cell growth and induces cell apoptosis via cell cycle arrest in human oral cancer SCC-4 cells. Environ Toxicol 2018;33(2):127-41.

17. Liang CQ, Fu YM, Liu ZY, Xing BR, Jin Y, Huang JL. The effect of miR-224 down-regulation on SW80 cell proliferation and apoptosis and weakening of ADM drug resistance. Eur Rev Med Pharmacol Sci 2017;21(21):5008-16.

18. Yu LM, Wang WW, Qi R, Leng TG, Zhang XL. MicroRNA-224 inhibition prevents progression of cervical carcinoma by targeting PTX3. J Cell Biochemi 2018;119(12):10278-90.

19. Yang C, Yuan W, Yang X, Li P, Wang J, Han J, et al. Circular RNA circ-ITCH inhibits bladder cancer progression by sponging miR-17/miR-224 and regulating p21, PTEN expression. Mol Cancer 2018;17(1):1-2.

20. Ma C, Wang X, Yang F, Zang Y, Liu J, Wang X, et al. Circular RNA hsa_circ_0004872 inhibits gastric cancer progression via the miR-224/Smad4/ADAR1 successive regulatory circuit. Mol Cancer 2020;19(1):1-21.

21. Yu X, Wang Y, Tao S, Sun S. Geniposide plays anti-tumor effects by down-regulation of microRNA-224 in HepG2 and Huh7 cell lines. Exp Mol Pathol 2020;112:104349. 